\title{
The role of neurotropism in psychiatric patients with COVID-19
}

\author{
Ailyn D. Diaz ${ }^{1} \cdot$ Raman Baweja' ${ }^{10}$ \\ Received: 27 June 2020 / Accepted: 17 September 2020 / Published online: 27 September 2020 \\ c) Springer-Verlag GmbH Germany, part of Springer Nature 2020
}

Coronavirus 2019 (COVID-2019) has a significant negative psychological impact on psychiatric patients as compared to healthy controls with higher rates of posttraumatic stress disorder (31.6\%), moderate to severe anxiety (23\%), depressive symptoms (22.4\%), and suicidal ideations (11.8\%) [1]. Psychiatric patients are especially vulnerable for infection with severe acute respiratory syndrome coronavirus-2 (SARS-CoV-2), which causes the respiratory disease state known as COVID-19 [2]. Medical comorbidities, such as diabetes mellitus and hypertension, magnify the risk of infection in the psychiatric population [1,2]. Factors such as impaired decision making, general psychomotor retardation, and poor adherence to social distancing can contribute to the susceptibility of infection [2]. Moreover, psychiatric patients might not be aware of their personal space or be adherent to self-protection methods, such as handwashing or wearing masks. This poor adherence is especially dangerous due to the mode of transmission of SARS-CoV-2; it spreads mainly through respiratory inhalation of aerosolized droplets through human-to-human spread via asymptomatic incubation carriers [3]. As the viral respiratory droplets are inhaled, it can spread to the lower respiratory tract causing dyspnea and, in some cases, attacking the central nervous system [4, 5]. Due to the role of neurotropism of SARS-CoV-2, the psychiatric complications of COVID-19 and the associated immunoreactivity of the viral illness, should be evaluated by clinicians since exposed psychiatric patients can potentially display an exacerbation of psychosis and mood symptoms.

The complications of COVID-19 in the central nervous systems are not uncommon and most likely caused by the invasive nature of the virus on neurons [4]. In a recent retrospective study of 214 patients in China with lab-confirmed COVID-19, 34\% displayed neurological manifestations, including an array of symptoms ranging from dizziness,

Raman Baweja

rbaweja@pennstatehealth.psu.edu

1 Department of Psychiatry and Behavior Health, Penn State University College of Medicine, 500 University Drive, H073, Hershey, PA 17033-0850, USA ataxia, impaired consciousness to acute cerebrovascular disease [5]. An earlier study during the initial outbreak in China showed that patients exhibited other neurological manifestations, such as headaches, nausea, and fatigue [3]. Despite known clinical and empirical research on neurological symptoms, there is limited knowledge of psychiatric complications with the disease.

In general, coronaviruses possess a positive-sense single-stranded RNA with an outer envelope and a crown-like shape [2, 3]. SARS-CoV-2 is one of the seven known coronaviruses to affect humans along with $\mathrm{HCo}-\mathrm{V}-229 \mathrm{E}, \mathrm{HCoV}-$ OC43. HCoV-NL63, HCoV-HKU1, which causes mild upper respiratory symptoms [4]. SARS-CoV and MERS$\mathrm{CoV}$ are beta-coronaviruses, which like SARS-CoV-2, can cause neurotropism and severe pathology, including pneumonia and death $[3,4]$. It is important to note that SARS$\mathrm{CoV}-2$ binds to angiotensin-converting enzyme-2 (ACE-2), a transmembrane receptor present in the central nervous system and skeletal muscle system [5]. Also, the tissue distribution of ACE-2 plays an active role in the illness; ACE-2 binding sites are more prevalent in the lungs leading to dyspnea but distributed less in the central nervous system [5]. Based on this, SARS-CoV-2 can potentially cause neuronal damage leading to psychiatric complications.

It is postulated that multiple pathways lead to the neurotropism of SARS-CoV-2 in the central nervous system. The inhaled coronavirus can infiltrate the olfactory bulb directly extending to the brain $[3,6]$. This route can affect the structures closest to the olfactory bulb, including the limbic system, which might likely lead to an exacerbation of mood disorders in psychiatric patients. In the hematogenous route, the inhaled virus can disseminate through the blood circulation and breakthrough to the blood-brain barrier by binding to ACE-2 receptors in the capillary endothelium [4, 5]. Furthermore, this route might manifest an infectious and toxic encephalopathy [4], which can be expressed as clinical delirium in psychiatric patients.

SARS-CoV-2 can reach the central nervous system by binding to peripheral nerve terminals. Through retrograde transport, the virus can cross synaptic connections reaching 
the central nervous system [4, 5]. A systemic cytokine response of the immune system can lead to pro-inflammatory factors in the brain that could cause chronic inflammation and brain damage $[4,5]$. Other indirect mechanisms to be considered involve hypoxic brain injury [4], which could eventually lead to cognitive sequelae, such as impaired memory recall. After penetrating the central nervous system, a highly sensitive system to homeostatic change, the coronavirus is removed slowly by macrophages [4]. This delayed response can cause a prevalent inflammatory state conducive to worsened symptoms of depression.

Seropositivity tests also demonstrate that coronavirus exposure may be a comorbid risk factor for patients with psychiatric illness, specifically psychosis and mood disorders [6, 7]. In 106 psychiatric patients who had a recent episode of psychosis, patients exposed to coronavirus showed a worsened onset of psychotic symptoms compared to controls without a psychiatric history [6]. Although patients exposed to coronavirus displayed a more significant antibody response for both mood and psychosis, a continuous elevation of IgG was found in those patients with schizophrenia-spectrum disorders compared to mood disorders [6]. The coronavirus types tested were not highly pathogenic (HCoV-229-E, HCoV-OC43. HCoV-NL63, HCoV-HKU1) compared to SARS-Cov-2, which appears to attack the most severe patients neurologically [4-6]. Another seropositivity study showed that coronavirus specifically HCoV-NL63 is associated with mood disorders but not necessarily the episode, major depressive or mania. Furthermore, there was no association between coronavirus and a history of suicidal attempt [7].

There is also accumulating evidence, which suggests that COVID-19 increases the risk of depressive and anxiety symptoms among healthy individuals [1]. Given that mood disorders are often under-diagnosed, it can be challenging for the clinicians to distinguish the cases in which COVID-19 exacerbated their mental illness from those in which COVID-19 itself caused the onset of mental illness. A large epidemiological study is needed to evaluate the further impact of COVID-19 on mental health.

With the advent of a serological assay test, it is essential to consider if SARS-CoV-2 will cause a similar acute immunoreactive response in psychiatric patients, specifically those with a history of psychosis or mood disorder. A serological assay could also provide further input as to the infectivity of the virus in the community. It can be useful in tracking cases of affected psychiatric patients to best study any exacerbation of their symptoms. Mental health professionals should be aware of the psychiatric implications of SARS-CoV-2 since there is an overlap of viral symptoms with the illness, such as low energy and fatigue, which also occurs with a major depressive episode. This awareness gains importance in those patients with mild COVID-19 symptoms who have not been tested through a serological assay. Furthermore, the neurotropism of the virus can lead to longstanding changes hence longitudinal studies are warranted to best understand the psychiatric repercussion of the illness. In summary, it is conjectured that coronaviruses affect psychiatric patients through neurotropism and a viral immune response, causing a susceptibility in the central nervous system, and leading to an exacerbation of psychosis and mood disorder.

\section{Compliance with ethical standards}

Conflict of interest Drs. Diaz and Baweja report no biomedical financial interests or potential conflicts of interest.

\section{References}

1. Hao F, Tan W, Jiang L, Zhang L, Zhao X, Zou Y et al (2020) Do psychiatric patients experience more psychiatric symptoms during COVID-19 pandemic and lockdown? A case-control study with service and research implications for immunopsychiatry. Brain Behav and Immun. 87:100-106

2. Zhu Y, Chen L, Ji H, Xi M, Fang Y, Li Y (2020) The risk and prevention of novel coronavirus pneumonia infections among inpatients in psychiatric hospitals. Neurosci Bull 36:299-302

3. Guan WJ, Ni ZY, Hu Y, Liang WH, Ou CQ, He JX et al (2020) Clinical characteristics of coronavirus disease 2019 in China. N England J Med 382(18):1708-1720

4. Wu Y, Xu X, Chen Z, Duan J, Hashimoto K, Yang L et al (2020) Nervous system involvement after infection with COVID-19 and other coronaviruses. Brain Behav and Immun 87:18-22

5. Mao L, Jin H, Wang M, Hu Y, Chen S, He Q et al (2020) Neurologic manifestations of hospitalized patients with coronavirus disease 2019 in Wuhan. China JAMA Neurol 77(6):683-690

6. Severance EG, Dickerson FB, Viscidi RP, Bossis I, Stallings CR, Origoni AE et al (2011) Coronavirus immunoreactivity in individuals with a recent onset of psychotic symptoms. Schizophr Bull 37(1):101-107

7. Okusaga O, Yolken RH, Langenberg P, Lapidus M, Arling TA, Dickerson FB et al (2011) Association of seropositivity for influenza and coronaviruses with history of mood disorders and suicide attempts. J Affect Disord 130(1-2):220-225 